\title{
Aplicación clínica de la técnica percutánea en la tenosinovitis estenosante de los flexores de la mano
}

\author{
M. Brotat ${ }^{\mathrm{I}}$, C. Simón Pérez ${ }^{\mathrm{I}}$, B. García Medrano ${ }^{\mathrm{I}}$, L. García Flórez , \\ M. Á. Martín Ferrero ${ }^{1}$,M.F. Muñoz Moreno ${ }^{2}$. \\ 'Servicio de Traumatología y Cirugía Ortopédica. Hospital Clínico Universitario de Valladolid. España. \\ 2Sección de Metodología y Bioestadística Laboratorio de Biomédicas en Investigación Clínica \\ CBIC. Hospital Clínico Universitario de Valladolid. España
}

\begin{abstract}
Resumen: Introducción: La tenosinovitis estenosante de los flexores de la mano es una patología que conduce al bloqueo de la polea de flexión proximal del dedo. Aunque el tratamiento inicial es conservador, si éste no es efectivo, está indicado el tratamiento quirúrgico mediante liberación abierta o percutánea.

Material y método: Estudio descriptivo retrospectivo de 116 pacientes intervenidos de dedo en resorte en el HCUV (enero 2005- diciembre 2007), seguimiento medio 36 meses. Análisis de datos con el $91 \%$ de los pacientes ( 105 pacientes) con un total de 182 dedos afectos realizando comparación de ambas técnicas.

Resultados: Edad media varones 53.70 años y la de las mujeres 53.95 . El 34\% de las manos intervenidas no presentan otras patologías asociadas. Primer dedo más afectado, seguido del tercero y cuarto. Se realizó técnica percutánea en el $42 \%$ de los pacientes, abierta en el $53 \%$ y ambas en el $5 \%$ del total. Destacan los problemas con la cicatriz en la tenolisis abierta. Técnica percutánea permite reincorporación a la vida laboral 8 días antes.

Discusión: La liberación percutánea de la polea Al es una alternativa a la liberación abierta por su bajo coste y por permitir una rápida reincorporación del paciente a su vida laboral. Gracias a estudios experimentales en cadáver, se obtienen referencias topográficas que aportan mayor seguridad, permitiendo ampliar el uso de esta técnica que ha demostrado grandes ventajas en el ámbito socio-económico y laboral.
\end{abstract}

Palabras claves: dedo en resorte, tenolisis percutánea, polea AI, tenosinovitis estenosante.

\section{Clinical applications of percutaneous trigger finger release}

\begin{abstract}
Introduction:Trigger finger leads to block at the proximal pulley of the finger. Although the initial treatment is conservative, if it is not effective, surgery is indicated by open or percutaneous tenolysis is indicated.

Material and method: Retrospective descriptive study of II6 patients surgery for trigger finger in HCUV (January 2005 -December 2007), mean follow-up of 36 months. Data analysis with $91 \%$ of patients ( $80 \%$ women, $20 \%$ men) with a total of 182 fingers affected comparing the two techniques.

Results: Male average age 53.7 years and for women 53.9. Thirty-four percent of operated hands have no other pathologies associated. First finger is the most affected, followed by the third and the fourth. Percutaneous technique was performed in $42 \%$ of patients, open technique in $53 \%$ and both in $5 \%$ of the total. Highlight problems with the scar on the open tenolysis. Percutaneous technique allows re-employment life eight days before the open technique.

Discussion: the percutaneous technique is an alternative to open release due to its low cost and because it allows a quick return of the patient to their working lives. Experimental studies get topographical references that provide greater security. This technique has shown great advantages.
\end{abstract}

Key words: Trigger finger, percutaneous technique, AI pulley.

La tenosinovitis estenosante de los flexores de la mano o dedo en resorte es una patología que conduce al bloqueo de la polea de flexión proximal del dedo., ${ }^{1,2}$ Es relativamente frecuente y puede afectar a personas en su etapa activa relacionándose con factores del ámbito laboral.

Se estima una incidencia de 28 casos por 100.000 habitantes por año. La etiología es controvertida habiéndose barajado un origen congénito y adqui- rido. Puede estar relacionado con microtraumatismos repetitivos que se producen en distintas actividades como la costura o trabajos artesanos ${ }^{2}$, hoy en día se asocia el antecedente laboral con una predisposi-
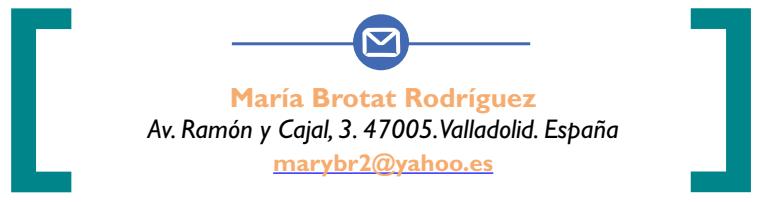
ción del sujeto a padecer enfermedades inflamatorias y reumatismos en las manos aunque algunos autores continúan defendiendo el origen adquirido como única opción ${ }^{3}$.

El dedo en resorte aparece con mayor frecuencia en el dedo pulgar, medio o anular de la mano dominante (generalmente la derecha) de las mujeres, mayores de 50 años, que padecen diabetes mellitus, hipotiroidismo, insuficiencia renal, gota, amiloidosis o artritis reumatoide, síndrome del túnel del carpo, contractura de Dupuytren y otras tendinitis (Quervain o epicondilitis lateral) $)^{3}$. Es una patología infrecuente en niños, con una incidencia del $0.05 \%$ al $0.3 \%$ representando un $2 \%$ de todas las anomalías congénitas del miembro superior pediátrico tratándose del procedimiento quirúrgico más frecuente que se realiza en la mano del niño ${ }^{5}$.

La resolución espontánea del dedo en resorte de larga evolución no suele producirse y la mayor complicación es la rigidez articular.

El objetivo del tratamiento en el dedo en resorte es eliminar el bloqueo y el dolor y así permitir un movimiento completo del dedo sin molestias 4 .

Se han descrito distintos tratamientos de tipo conservador como la inmovilización con férula de yeso, el uso de antinflamatorios orales, la electromagnetoterapia $^{6,7}$ o la infiltración con corticoides ${ }^{8}$. De todas formas el tratamiento quirúrgico sigue siendo necesario en los casos en los que el tratamiento conservador no es efectivo siendo la tenolisis abierta y la liberación percutánea las dos técnicas de elección.

Aunque el éxito de la técnica abierta se estima prácticamente en un $100 \%$ se han descrito numerosas complicaciones que motivan la búsqueda de nuevos procedimientos ${ }^{5,9}$.

El objetivo es comprobar las diversas ventajas de la tenolisis percutánea frente a la tenolisis convencional en el tratamiento de la tenosinovitis estenosante de los flexores de los dedos, diferenciando el dedo afectado así como la repercusión en la vida laboral de los pacientes intervenidos por ambas técnicas.

\section{MATERIALY MÉTODO}

Se analizan 116 pacientes diagnosticados de tenosinovitis estenosante de los flexores de la mano intervenidos quirúrgicamente en el Hospital Clínico Uni- versitario de Valladolid entre enero 2005 y diciembre del 2007 con un periodo medio de seguimiento de 36 meses.

Los criterios de inclusión son pacientes mayores de 18 años intervenidos quirúrgicamente de dedo en resorte con sintomatología superior a 3 meses hasta años de evolución que no mejoran con el tratamiento conservador (AINES, infiltraciones, rehabilitación), diferenciando patología del primer dedo y de los dedos largos, y pacientes intervenidos previamente por la misma patología en el mismo o en diferentes dígitos.

Se excluyen del estudio aquellos pacientes diagnosticados de dedo en resorte grado I de la clasificación de Eastwood y de origen congénito.

Se analizan diversas variables:

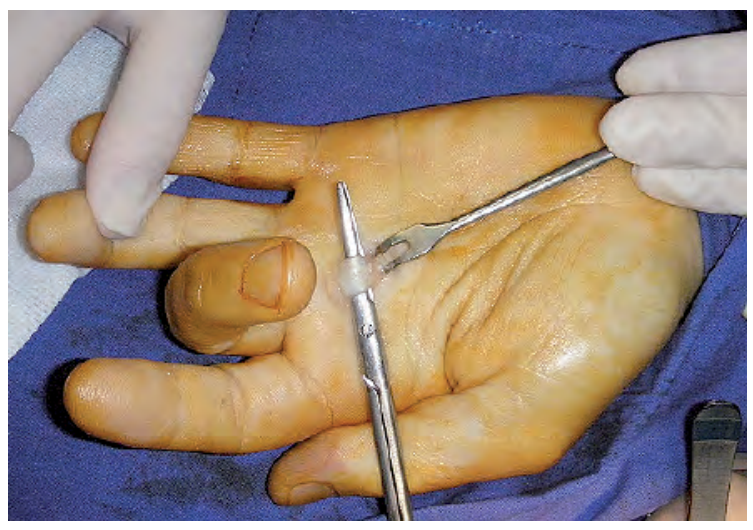

Figura. I. Tenolisis convencional a cielo abierto.

I. Técnica quirúrgica (localización de la incisión, lesiones tendinosas por la aguja, angulación adecuada de la aguja).

2. Comparación de ambas técnicas: cicatrización, posibles complicaciones como las infecciones post-operatorias o dehiscencias de la herida, tiempo de incapacidad y de reincorporación a la vida laboral.

3. Epidemiología (edad, sexo, etiología, clínica habitual, técnicas diagnósticas).

4. Comparación entre el pulgar y los dedos largos tanto en la técnica quirúrgica como en las complicaciones.

En la técnica abierta se realiza una incisión trasversal, se localizan y se separan las estructuras vasculonerviosas, se identifica la polea realizando apertura lon- 
gitudinal de la misma y tenolisis de los flexores. Tras haber comprobado que la liberación del tendón es completa, se sutura la piel y se aplica un pequeño vendaje compresivo. (2) Figura I.

La otra técnica utilizada es la tenolisis percutánea en la que se sitúa al paciente mostrando la cara volar de la mano con el dígito en extensión. Se introduce una aguja 16G perpendicular a la polea Al y con movimientos de oscilación longitudinales se secciona la polea intentando no dañar el tendón. Para ello se flexiona y se extiende el dedo observando el movimiento de la aguja, si ésta se mueve significa que está situada en el tendón por lo que se debe retirar levemente para seccionar sólo la polea. La incisión debe ser realizada exactamente sobre la línea media del metacarpo y rigurosamente paralela a esta línea ya

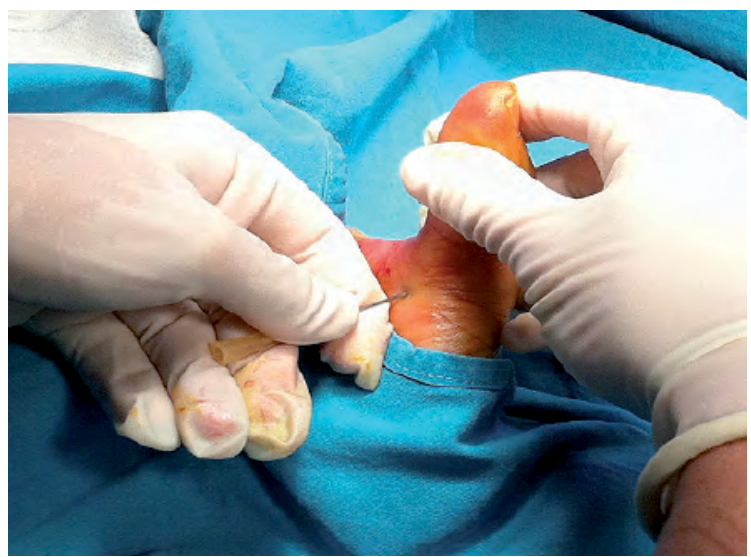

Figura 2. Tenolisis percutánea.

que de esta forma es más difícil dañar el paquete neurovascular. Aunque con una sola punción se puede seccionar toda la polea, en ocasiones es necesario realizar más punciones a nivel distal y proximal para conseguir la liberación completa de los tendones flexores. Hay que insistir mucho en la movilización de dedo en las primeras 24-48 horas por lo que las revisiones post-operatorias son importantes. (2) Figura 2.

Al realizar el análisis estadístico las variables cuantitativas se presentan con la media y la desviación típica y las cualitativas según su distribución de frecuencias. Mediante el test chi-cuadrado de Pearson, se ha analizado la asociación de las variables cualitativas. En el caso de que el número de celdas con valores esperados menores de 5 sea mayor de un 20\%, se ha utilizado el test exacto de Fisher o el test Razón de verosimilitud para variables con más de dos categorías.
Las comparaciones de los valores cuantitativos según la técnica, se ha realizado mediante la prueba $T$ de Student para muestras. Los datos han sido analizados con el programa estadístico SPSS versión 20.0 para Windows, aquellos valores de $p \leq 0,05$ han sido considerados estadísticamente significativos.

\section{RESULTADOS}

Se realiza el análisis de datos con 105 pacientes (91\%) descartando aquéllos no localizados, obteniendo un total de 182 dedos afectos.

Se obtiene una muestra de 84 mujeres (79\%) y 21 varones (21\%). El rango de edad de las mujeres afectadas oscila entre 28 y 74 años con una edad media de 53.95 años, el rango correspondiente a los hombres se encuentra entre 18 y 77 años con una edad media de 53.70.

Ciento dos pacientes (97.10\%) tienen afectada su mano dominante.

\section{Distribución según profesión}

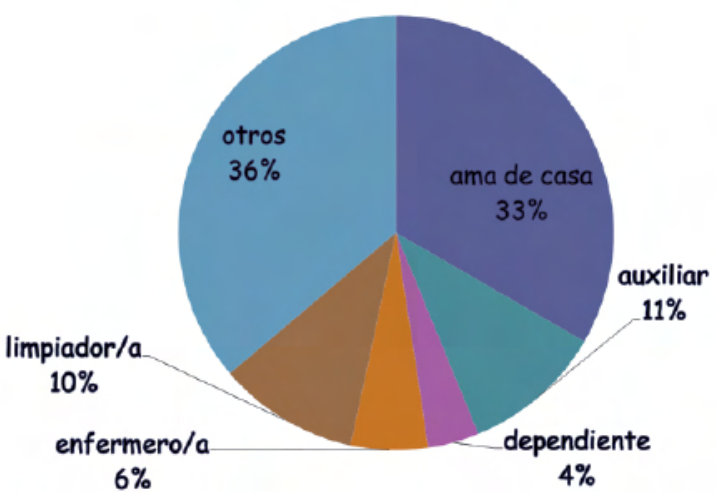

Figura 3. Distribución según profesión.

Las profesiones de los pacientes afectados corresponden a trabajos en los que se realizan tareas manuales. El 32\% son amas de casa seguidas en frecuencia por auxiliares, limpiadores/as y enfermeros/as. En el grupo correspondiente a otros se han incluido profesiones como mecánico, frutero/a, conductor/a, administrativo/a, cocinero/a u hostelero/a. (s) Figura 3.

Aunque se analizan 105 pacientes, el total de dedos en resorte asciende a 182 . A cuarenta y cuatro 


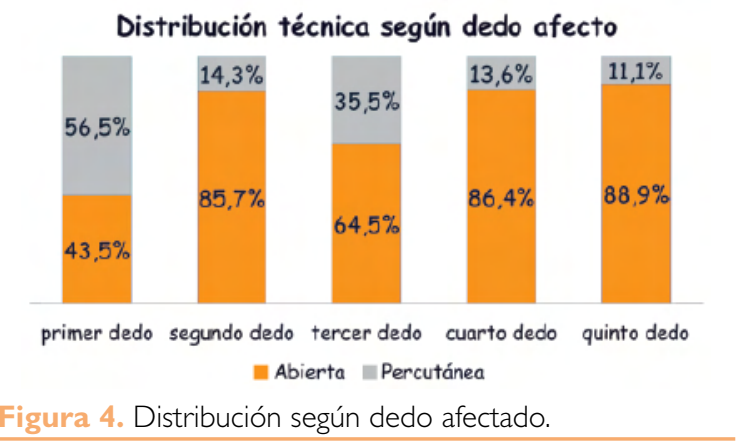

pacientes (42\%) se les realizó la técnica percutánea, a 56 pacientes (53\%) la técnica abierta y a 5 pacientes (5\%) ambas técnicas, bien por tener varios dedos afectos o por utilizar una técnica diferente en caso de recidiva. Figura 4.

Cuarenta y cuatro pacientes tenían más de un dedo afectado. En este grupo de pacientes, treinta (28.6\%) fueron intervenidos mediante tenolisis abierta, frente a 14 (13.6\%) intervenidos mediante técnica percutánea $(p=0,074)$ El primer dedo es el más afectado seguido en frecuencia del tercer y cuarto dedo. () Tabla I

Sólo el 34\% de las manos intervenidas no presentan otras patologías asociadas. Al analizar los datos se observa que 38 pacientes (35.70\%) a los que se les realizó la técnica percutánea padecían de síndrome del túnel del carpo frente a 62 (59.10\%) que se intervinieron mediante la técnica abierta $(p=0,020)$. En ocasiones, además, se asocian a otras patologías como rizartrosis, enfermedad de Dupuytren o tendinitis de Quervain.

El tiempo medio que los pacientes tardaron en acudir a la consulta desde que comenzaron a tener los primeros síntomas es de 200 días (6.7 meses) por ello no es de extrañar que la totalidad de los pacientes se encontraran en los grados 3 y 4 de la clasificación de Eastwood.

Previamente a la cirugía, 87 pacientes (83\%) realizaron tratamiento conservador como el uso de ortesis, la utilización de infiltraciones o la administración de AINES, bien como tratamiento único o en combi- nación, no encontrando diferencias estadísticamente significativas.

Todos ellos se operaron en régimen de Cirugía Mayor Ambulatoria sin pauta antibiótica previa a la cirugía, a excepción de los pacientes que presentaban patologías de carácter sistémico asociadas. (2) Tabla II.

En la mitad de los casos la anestesia fue local y en la otra mitad loco-regional. En el 54.50\% $(n=24)$ de la técnica percutánea no se asociaron otros procedimientos quirúrgicos $(p=0,008)$ frente a un $28.60 \%$ $(n=16)$ de la técnica abierta.

No se han encontrado diferencias estadísticamente significativas en las complicaciones según la técnica utilizada. Sin embargo, es interesante destacar que los problemas que proporciona la cicatriz con la técnica abierta son un $15.40 \%$ mayor que los causados en la tenolisis percutánea con un p- valor de 0.056 . (d) Tabla III.

Las personas que tienen afectado el primer dedo recuperan la movilidad completa en una media de 14 días (rango comprendido entre 7 y 25) sin ser necesaria la rehabilitación, independientemente de la técnica quirúrgica utilizada. Sin embargo, los pacientes intervenidos de los dedos largos tardan una media de 18 días (rango comprendido entre 10 y 30).

Un parámetro importante que analizamos es el tiempo que el paciente tarda en reincorporarse a su vida laboral en función de la técnica quirúrgica utilizada. Los pacientes intervenidos mediante tenolisis percutánea se incorporaron a los 36 días de la intervención, 8 días antes que aquellos intervenidos mediante técnica abierta, aunque no tiene valor estadísticamente significativo.

Al preguntar a los pacientes sobre la posibilidad de volver a operarse el $89.50 \%$ de los pacientes (94) estarían dispuestos a hacerlo. El grado de satisfacción de los pacientes es muy elevado, 92 pacientes (88\%) intervenidos mediante la técnica percutánea dan puntuaciones por encima de 7 y, en la misma situación, se obtiene un $90 \%$ en la técnica abierta.

\section{TABLA I - Afectación de dígitos.}

\begin{tabular}{cccccc} 
TOTAL & Primer dedo & Segundo dedo & Tercer dedo & Cuarto dedo & Quinto dedo \\
\hline 182 & 86 & 10 & 43 & 30 & 13 \\
\hline
\end{tabular}




\section{TABLA II - Procesos QUiRúrgicos asociados.}

\section{ASOCIACIÓN DE OTROS PROCESOS QUIRÚRGICOS}

Neurolisis
Artroplastia
Neurolisis y artroplastia
Neurolisis y otros
Otros
Nada

\section{DISCUSIÓN}

En nuestro estudio se observa que el $80 \%$ de los pacientes son de sexo femenino con una media de edad de 53.95 años, no encontrando diferencias respecto a otros estudios publicados ${ }^{10,11}$.

Hoy en día el origen del dedo en resorte se relaciona con profesiones en las que se realizan actividades manuales ${ }^{3}$ como ama de casa, auxiliar, limpiador/a o enfermero/a que, en nuestro caso, son las más pre- valentes. La presencia de otro tipo de patologías asociadas es importante, destacando en nuestro estudio que un $47.62 \%$ del total de pacientes padecen de síndrome del túnel carpiano asociado en muchas ocasiones a otras tenosinovitis estenosantes como tendinitis de Quervain, patologías como rizartrosis y enfermedad de Dupuytren o enfermedades sistémicas como Diabetes Mellitus e hipotiroidismo, como apoyan varios autores ${ }^{3}$.

Se han descrito numerosos tratamientos de tipo conservador en pacientes no complicados que presen-

\section{TABLA III - Complicaciones de AMBAS tÉCNICAS.}

\begin{tabular}{|l|c|c|c|c|}
\hline \multirow{2}{*}{ COMPLICACIONES } & \multicolumn{2}{|c|}{ Técnica abierta } & \multicolumn{2}{c|}{ Técnica percutánea } \\
\hline Déficit de movimiento & Frecuencia & Porcentaje & Frecuencia & Porcentaje \\
\hline Déficit de movimiento + alteraciones de la & 2 & $3,57 \%$ & 2 & $4,54 \%$ \\
sensibilidad & 1 & $1,79 \%$ & 2 & $4,54 \%$ \\
Alteraciones de la sensibilidad & 0 & $0 \%$ & 4 & $9,09 \%$ \\
Dolor & 4 & $7,14 \%$ & 4 & $9,09 \%$ \\
Dolor + alteraciones de la sensibilidad & 1 & $1,79 \%$ & 1 & $2,27 \%$ \\
Dolor + déficit de movimiento & 1 & $1,79 \%$ & 0 & $0 \%$ \\
\hline Dolor + recidiva & 0 & $0 \%$ & 1 & $2,27 \%$ \\
\hline Problemas cicatriz & 16 & $28,57 \%$ & 0 & $0 \%$ \\
\hline Problemas cicatriz + alteración de & 3 & $6,82 \%$ & 0 & $0 \%$ \\
la sensibilidad & 2 & $5,57 \%$ & 0 & $0 \%$ \\
Problemas cicatriz + déficit de movimiento & 1 & $1,79 \%$ & 4 & $9,09 \%$ \\
\hline Recidiva & 1 & $1,79 \%$ & 0 & $0 \%$ \\
Recidiva + déficit de movimiento & 0 & $0 \%$ & 0 & $0 \%$ \\
\hline Lesiones vasculares & 0 & $0 \%$ & 0 & $0 \%$ \\
\hline Lesiones tendinosas & 24 & $42,85 \%$ & 22 & $50 \%$ \\
\hline Nada & $\mathbf{5 6}$ & $\mathbf{1 0 0} \%$ & $\mathbf{4 4}$ & $\mathbf{1 0 0}$ \\
\hline TOTAL & & &
\end{tabular}


tan poco tiempo desde el comienzo de los síntomas. Algunos autores han sugerido como medida preventiva el empleo de almohadillas palmares que distribuyan la fuerza en un área amplia y de herramientas más anchas para disminuir el grado de flexión de los dedos". Otros apoyan el uso de diferentes tipos de ortesis que inmovilizan las articulaciones metacarpofalángicas en ortoposición o ligera flexión dejando libres las interfalángicas o que inmovilizan sólo las interfalángicas distales. Nuestra experiencia no es muy amplia en este tipo de tratamientos ya que únicamente un $2 \%$ de nuestros pacientes utilizaron ortesis previa a la cirugía.

Las inyecciones de corticoides son un tratamiento controvertido, mientras que Benson y Ptaszek hablan de una tasa de éxito del 60\% tras una infiltración única' ${ }^{12}$ otros autores afirman que en el $66 \%$ de los casos no es suficiente con una sola inyección y que finalmente la mayoría de los casos recidivan, siendo necesario el tratamiento quirúrgico ${ }^{13}$. Algunas publicaciones afirman que en pacientes jóvenes y diabéticos la tasa de fracaso es mucho más elevada no apoyando su utilización ${ }^{14}$. En nuestra serie sólo a un 10\% de los pacientes se les realizaron infiltraciones, la mayoría de las veces acompañadas de tratamiento analgésico y rehabilitador. La totalidad de estos pacientes necesitaron cirugía posteriormente tras una media de tres meses de efectividad de la infiltración.

Sin embargo, a pesar de que el tratamiento conservador es el de primera elección en el dedo en resorte, en la mayoría de los casos existen estudios que afirman que los tratamientos conservadores prolongan el retorno al trabajo y la duración de la sintomatología, y que, cuando es superior a los 6 meses y presenta localizaciones múltiples, disminuye los resultados quirúrgicos satisfactorios al $44 \%{ }^{15}$.

La técnica tradicional de liberación de los tendones flexores ha sido la técnica abierta. A partir de 1958, a raíz de los trabajos realizados por Lorthior ${ }^{5}$, la técnica percutánea es una alternativa a la liberación abierta tradicional aunque su relación riesgo-beneficio es muy discutida.

En nuestra serie, así como en diversas publicaciones, aparece la tasa de éxito similar tanto en la técnica abierta como en la percutánea ${ }^{16}$. Sin embargo, en el caso de la técnica tradicional se han documentado complicaciones como la distrofia simpático-refleja, la infección o lesiones nerviosas. En nuestro caso, las complicaciones más frecuentes fueron los problemas relacionados con la cicatriz: dolor, hipersensibilidad $y$, en algunos ocasiones, fibrosis cicatricial que puede condicionar la flexo-extensión.

En el caso de la tenolisis percutánea no se han descrito complicaciones graves, con la excepción de un caso publicado con un déficit sensitivo radial del primer dedo. ${ }^{17}$ La falta de visualización en esta técnica aumenta el riesgo de lesionar estructuras adyacentes como el paquete neurovascular, los tendones flexores - la polea A2 además de añadir dificultad a la liberación completa de la polea provocando un mayor número de casos de recidiva. ${ }^{18}$

Es importante destacar la relación del paquete neurovascular con la polea $\mathrm{Al}$ en el primer, segundo y quinto dedos provocando un mayor riesgo a la hora de realizar la técnica percutánea. Sin embargo, en nuestro estudio, el $68.62 \%$ de las intervenciones percutáneas se han realizado en el primer dedo ya que la situación de la polea Al es muy superficial en este dígito, sin haber observado mayor número de complicaciones.

Algunos autores contraindican la cirugía percutánea en el dedo en resorte grado IV o cuando existe una tenosinovitis florida, sin embargo, al igual que estudios realizados por Tanaka y Eastwood ${ }^{19,20}$, en nuestro estudio no se constataron complicaciones mayores afirmando más del $90 \%$ de los pacientes que volverían a operarse.

Estudiando diversos síntomas como la rigidez o el dolor nocturno, hemos encontrado mayores porcentajes de mejoría en la técnica percutánea. Es verdad, que en muchas ocasiones, la tenolisis se ha acompañado de otras técnicas quirúrgicas como la neurolisis del mediano o la colocación de la realización de artroplastia que posiblemente hayan condicionado el resultado final y la valoración del paciente.

Algunos autores afirman que la técnica abierta es una técnica más cruenta, de lenta recuperación funcional y con una reincorporación tardía a las actividades cotidianas mientras que la técnica percutánea produce una disminución significativa de la intensidad y duración del dolor postquirúrgico y permite un retorno inmediato al trabajo ${ }^{15}$ como confirmamos en nuestro estudio.

Una ventaja muy importante de la tenolisis percutánea es su bajo coste ya que únicamente es necesaria una aguja, mientras que la tenolisis abierta requiere 
de una mayor cantidad de instrumental, un aparato de isquemia, un ayudante y una sutura ${ }^{16}$.

Los estudios en cadáver son básicos para establecer las referencias anatómicas y poder utilizar los instrumentos quirúrgicos en la dirección adecuada. ${ }^{21}$ Existen autores que, incluso, señalan la utilización de ultrasonidos como guía para evitar complicaciones de carácter neurovascular $22,23$.

\section{CONCLUSIONES}

La tasa de éxito es similar en ambas técnicas quirúrgicas.

La complicación más frecuente en la técnica tradicional son los problemas relacionados con la cicatriz mientras que, en el caso de la tenolisis percutánea es la falta de liberación completa, sobre todo en el inicio de la curva de aprendizaje del cirujano.

La tenolisis percutánea de la polea AI tiene un bajo coste y permite una rápida reincorporación del paciente a su vida laboral.

Los estudios anatómicos son importantes ya que buscan nuevos instrumentos quirúrgicos y referencias topográficas que aporten mayor seguridad a la liberación percutánea.

\section{CONFLICTOS DE INTERESES}

Los autores declaran no tener conflictos de intereses.

\section{BIBLIOGRAFÍA}

I. Llusá M, Merí A, Ruano D. Manual y atlas fotográfico de anatomía de aparato locomotor. Editorial Médica Panamericana; 2007.

2. Rohen, Yokochi. Atlas fotográfico de anatomía humana. Doyma libros. 1994.

3. Chaves Moreno, Allan. Tenosinovitis estenosante del tendón flexor. Medicina legal de Costa Rica. 2008; 25:59-65.

4. Marcos Fuentes L, Downey Carmona FJ, Tatay Díaz A, Macías Moreno E, Farrington Rueda D. Pulgar en resorte en niños. Comparación de resultados entre la liberación percutánea y la cirugía abierta. Rev Esp Cir Ortop Traumatol. 20 I 0;54:35 I-6.
5. Lorthioir J. Surgical treatment of trigger-finger by a subcutaneous method. J Bone Joint Surg Am. 1958:40:793-5.

6. Sosas Salinas U, Hernández Rodríguez A, Morfa Viamontes, F. Dedo en resorte por nódulo del tendón flexor: tratamiento mediante electromagnetoterapia en atención primaria. Rev Cubana Ortop Traumatol. 1997; I 1:25-30.

7. Brent S, Wilk K. Rehabilitación Ortopédica Clínica. $2^{a}$ Edición. Madrid: Elsevier España; 2007.

8. Peters-Veluthamaningal $C$, van der Windt DA, Winters JC, Meyboom-de Jong B. C. Corticosteroid injection for trigger finger in adults. Cochrane Database Syst Rev. 2009; I:CD0056 17

9. FuY, Huang PJ, Tien YC, Lu YM, Fu HH, Lin GT. Revision of incompletely released trigger fingers by percutaneous release: results and complications. J Hand Surg Am. 2006;31: I 288-91.th

10. Sánchez Martín, M. Traumatología y Ortopedia. Afecciones no traumáticas del codo, muñeca y mano. 2002:2:875.

I I. Campbell. Síndrome del túnel carpiano, síndrome del túnel cubital y tenosinovitis estenosante. Cirugía Ortopédica. $10^{\circ}$ Ed. 2005; $10^{\circ}$ Ed; vol 4; 37743775.

12. Benson LS, Ptaszek AJ. Injection versus surgery in the treatment of trigger finger. I Hand Surg Am. 1997;22(I): I 38-44.

13. Tallia A. Diagnostic and therapeutic injection of the wrist and hand region. American Physician. 2003;67:745-50.

14. Tamara D, R; Zurakowski, D; Blazar,P. Trigger finger: prognostic indicators of recurrence following corticosteroid injection. J Bone Joint Surg Am. 2008:90:1665-72.

15. Saldana MJ.Trigger digits diagnosis and treatment. J Am Acad Orthop Surg. $2001 ; 9: 246-52$.

16. Gilberts E, Beekman W, Stevens H, Wereldsma J. Prospective randomized trial of open versus percutaneous surgey for trigger digits. J Hand Surg. 200 I;26A:497-500.

17. Jongjirasiri Y.The results of percutaneous release of trigger digits by using full handle knife 15 degrees: an anatomical hand surface landmark and clinical study. J Med Assoc Thai. 2007;90: I 348-55.

18. Igualada Blázquez C, Rojo Manaute JM, Tabernero Fernández JM, Rodríguez Conde C, Del Cerro Gutiérrez M, Vaquero Martín J. Polectomía percutánea guiada por ECO. Parte I: descripción anatómica de las "zonas seguras". Trauma Fundación MAPFRE. 2003;21:136-140. 
19. Tanaka J, Muraji M, Negoro H,Yamashita H, Nacano T, Nacano K. Subcutaneous release of trigger thumb and fingers in 210 fingers. J Hand Surg. 1990; | 5B:463-5.

20. Eastwood DM, Gupta KJ, Johnson DP. Percutaneous release of the trigger finger: an office procedure. J Hand Surg. 1992;17A:1 I4-7.

21. Brotat M, Simón C, García Medrano B, García Flórez L, Martín Ferrero MA. Referencias anatómicas para la tenolisis percutánea de los flexores de la mano. Rev Iberamer Cir Mano. 20 I 4;42:4 I-46.

22. Chern TC, Jou IM, Yen SH, Lai KA, Shao CJ. Cadaver study of sonographically assisted percutaneous release of the Al pulley. Plast Reconstr Surg. 2005; | | 5:8 | I-22.

23. Paulius KL, Maguina P. Ultrasound-Assisted Percutaneous Trigger Finger Release: Is it Safe? Hand (NY). 2009:4:35-7. 